\title{
Multi-objective Scatter Search with External Archive for Portfolio Optimization
}

\author{
Khin Lwin ${ }^{1, *}$, Rong $\mathrm{Qu}^{1}$ and Jianhua Zheng ${ }^{2}$ \\ ${ }^{1}$ ASAP Research Group, School of Computer Science, University of Nottingham, NG8 1BB, Nottingham, UK \\ ${ }^{2}$ School of Computer Science, University of Nottingham, NG8 1BB, Nottingham, UK \\ *ktl@cs.nott.ac.uk
}

Keywords: Evolutionary Multi-objective Portfolio Optimization: Hybrid Metaheuristic: Multi-objective Scatter Search: Cardinality Constrained Portfolio Selection Problem: Mean-Variance Portfolio Optimization.

Abstract: The relevant literature showed that many heuristic techniques have been investigated for constrained portfolio optimization problem but none of these studies presents multi-objective Scatter Search approach. In this work, we present a hybrid multi-objective population-based evolutionary algorithm based on Scatter Search with an external archive to solve the constrained portfolio selection problem. We considered the extended meanvariance portfolio model with three practical constraints which limit the number of assets in a portfolio, restrict the proportions of assets held in the portfolio and pre-assign specific assets in the portfolio. The proposed hybrid metaheuristic algorithm follows the basic structure of the Scatter Search and defines the reference set solutions based on Pareto dominance and crowding distance. New Subset generation and combination methods are proposed to generate efficient and diversified portfolios. Hill Climbing operation is integrated to search for improved portfolios. The performance of the proposed multi-objective Scatter Search algorithm is compared with the Non-dominated Sorting Genetic Algorithm (NSGA-II), Strength Pareto Evolutionary Algorithm (SPEA-2) and Pareto Envelope-based Selection Algorithm (PESA-II). Experimental results indicate that the proposed algorithm is a promising approach for solving the constrained portfolio selection problem. Measurements by the performance metrics indicate that it outperforms NSGA-II, SPEA2 and PESA-II on the solution quality within a shorter computational time.

\section{INTRODUCTION}

The portfolio selection problem is concerned with the optimal allocation of a limited capital among available risky assets. The mean-variance (MV) model of Markowitz (Markowitz, 1952) (Markowitz, $1959)$ is a single period static portfolio model based on two criteria: to maximize the reward of a portfolio (measured by the mean of return), and to minimize the risk of the portfolio (measured by the variance of return). It is considered to be the first mathematical model that formalizes the concept of diversification and is widely used in finance. It helps risk-averse investors to construct desirable portfolios by taking into consideration of the trade-off between risk and expected return. With the above stated two conflicting objectives, the portfolio selection problem could be considered as a class of multi-objective optimization problems. As a consequence, a single unique solution that optimizes all the conflicting objectives hardly exists and instead there exists a set of solutions which are optimal in such a way that no other solutions in the search space are superior to them when all objectives are considered simultaneously. This set of solutions is known as non-dominated or Pareto optimal set.
Although Markowitz's MV model is the fundamental theory of Modern Portfolio Theory, direct application of this model is not of much practical uses mainly due to the fact that it is simplified with unrealistic assumptions. In many practical situations, constraints such as cardinality, quantity and pre-assignment constraints are required. The cardinality constraint imposes a limit on the number of assets in the portfolio either to simplify the management of the portfolio or to reduce transaction costs. The quantity constraint restricts the proportion of each asset in the portfolio to lie between the lower and upper bounds in order to avoid very small (or large) and unrealistic holdings. The pre-assignment constraint requires certain asset(s) to be included in the portfolio. These constraints are hard in the sense that they have to be satisfied at any time. In this work, Markowitz's MV model extended with cardinality, quantity and pre-assignment constraints is studied.

When the basic model is extended with such practical constraints, the problem transforms to an NPHard problem (Moral-Escudero et al., 2006) and exact methods are inadequate to solve the problem in an efficient way. In recent years, several researches have been conducted applying metaheuristics to the portfo- 
lio selection problem with real-world constraints. Although the portfolio selection problem involves two conflicting objectives, many researches in the literature have been performed with single objective approaches with aggregating function (Chang et al., 2000) (Lwin and Qu, 2013). Recently, there are an increasing number of multi-objective evolutionary approaches that have been studied. The main advantage of evolutionary multi-objective portfolio optimization is that an estimation of the efficient risk-return frontier can be searched in a single run as opposed to the single objective approaches with multiple runs. Several researches have been conducted to solve the portfolio optimization problem by adopting the evolutionary multi-objective approach (Branke et al., 2009) (Skolpadungket et al., 2007).

The aim of this paper is to apply the multiobjective scatter search to the mean-variance portfolio optimization problem. The rest of the paper is organized as follows. Section- 2 provides the mean-variance model with cardinality, quantity and pre-assignment constraints considered in this study. Section-3 presents the multi-objective Scatter Search algorithm with external archive and describes the details of its components. Section-4 is provided with numerical results and conclusion remarks are presented in Section-5.

\section{MULTI-OBJECTIVE CONSTRAINED PORTFOLIO OPTIMIZATION}

The portfolio optimization problem concerns the choice of an optimal set of assets to include in the portfolio and the distribution of investment among them. In the multi-criteria variant of portfolio optimization problem, the model is usually formalized as follows:

$$
\begin{gathered}
\min f_{1}=\sum_{i=1}^{N} \sum_{j=1}^{N} w_{i} w_{j} \sigma_{i j} \\
\max \quad f_{2}=\sum_{i=1}^{N} w_{i} \mu_{i} \\
\text { subject to } \sum_{i=1}^{N} w_{i}=1 \\
\sum_{i=1}^{N} s_{i} \leq K, \\
\varepsilon_{i} s_{i} \leq w_{i} \leq \delta_{i} s_{i}, \quad i=1, \ldots, N, \\
s_{i} \geq z_{i}, \quad i=1, \ldots, N \\
s_{i} \in\{0,1\}, \quad i=1, \ldots, N \\
z_{i} \in\{0,1\}, \quad i=1, \ldots, N
\end{gathered}
$$

where $N$ is the number of available assets, $\mu_{i}$ is the expected return of asset $i(i=1 \ldots N), \sigma_{i j}$ is the covariance between assets $i$ and $j(i=1 \ldots N ; j=1 \ldots N)$, and $w_{i}\left(0 \leq w_{i} \leq 1\right)$ is the decision variable which represents the proportion held of asset $i$. Eq(3) defines the budget constraint (all the money available should be invested) for a feasible portfolio. $K$ is the maximum number of invested assets in the portfolio, $s_{i}$ denotes whether asset $i$ is invested or not, $z_{i}$ denotes a binary value such that $z_{i}$ is set to one if asset $i$ is included in the pre-assigned set in the portfolio. If $s_{i}$ equals to one, asset $i$ is chosen to be invested and the proportion of capital $w_{i}$ lies in $\left[\varepsilon_{i}, \delta_{i}\right]$, where $0 \leq \varepsilon_{i} \leq \delta_{i} \leq 1$. Otherwise, asset $i$ is not invested and $w_{i}$ equals to zero.

\section{MULTI-OBJECTIVE SCATTER SEARCH WITH EXTERNAL ARCHIVE (MOSSwA)}

In this paper, a multi-objective Scatter Search with external archive has been developed for constrained portfolio selection problem. Scatter Search (SS) (Glover et al., 2000) is a population based metaheuristic that uses sets of solutions called the reference sets to combine its solutions to construct others. The method generates reference sets from a population of solutions. Then a subset of solutions is selected from the reference sets. The selected solutions are combined to generate new solutions. The generated solutions are enhanced by an improvement procedure. The result of the improved solution can motivate the updating of the reference sets. The proposed multi-objective Scatter Search with external archive (MOSSwA) consists of procedures namely, initial population creation, reference sets formation, subset generation, solution combination, improve solution, reference set update and archive update. The detailed procedure of the MOSSwA is provided in Figure-1.

\subsection{Solution Representation}

In our solution representation, two vectors of size $N$ are used to define a portfolio $p$ : a binary vector $s_{i}$, $i=1, \ldots, N$ denoting whether asset $i$ is included in the portfolio, and a real-value vector $w_{i}, i=1, \ldots, N$ representing the proportions of the capital invested in the assets.

\subsection{Initial Population Generation Method}

To generate a trial initial population, maximum $K$ different indexes (including all assets in the preassignment subset) are randomly selected and propor- 


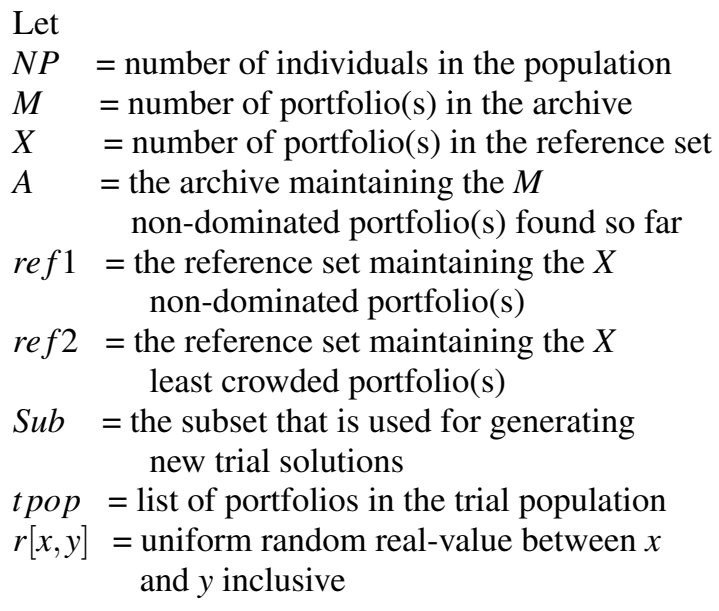

tions are assigned to those selected assets randomly. If the generated portfolio violates the budget and/or quantity constraints, such solution is corrected by the constraint handling techniques provided in Section3.9. Hence, all generated solutions in the trial population are feasible.

\subsection{Reference Set Formation}

The reference sets are initialized from an initial population composed of random solutions and they are updated by the improved and diverse solutions. Two reference sets are maintained: refl and ref2. The refl 1 and ref 2 sets maintain the best solutions and the least crowded solutions from the archive $(A)$ and the trial population(t pop) by Pareto optimal (Fonseca and Fleming, 1995) and the crowding distance (Deb et al., 2002) concepts respectively.

A solution $a$ is efficient (i.e., Pareto optimal) if there does not exist any solution $b$ such that $b$ dominates $a$. Solution $a$ is considered to dominate solution $b$ if and only if:

$$
\begin{aligned}
& f_{1}(a) \leq f_{1}(b) \text { AND } f_{2}(a)>f_{2}(b) \\
& \text { OR } \\
& f_{2}(a) \geq f_{2}(b) \text { AND } f_{1}(a)<f_{1}(b)
\end{aligned}
$$

The crowding distance is used to measure the approximate density of solutions toward each other in a population. The crowding distance of a portfolio $p$ is calculated by taking the average distance of the two portfolios taken one from either side of $p$. (Deb et al., 2002)

\subsection{Subset Generation Method}

This method selects a subset of solutions from the reference set to create a subset $(S u b)$. This generated

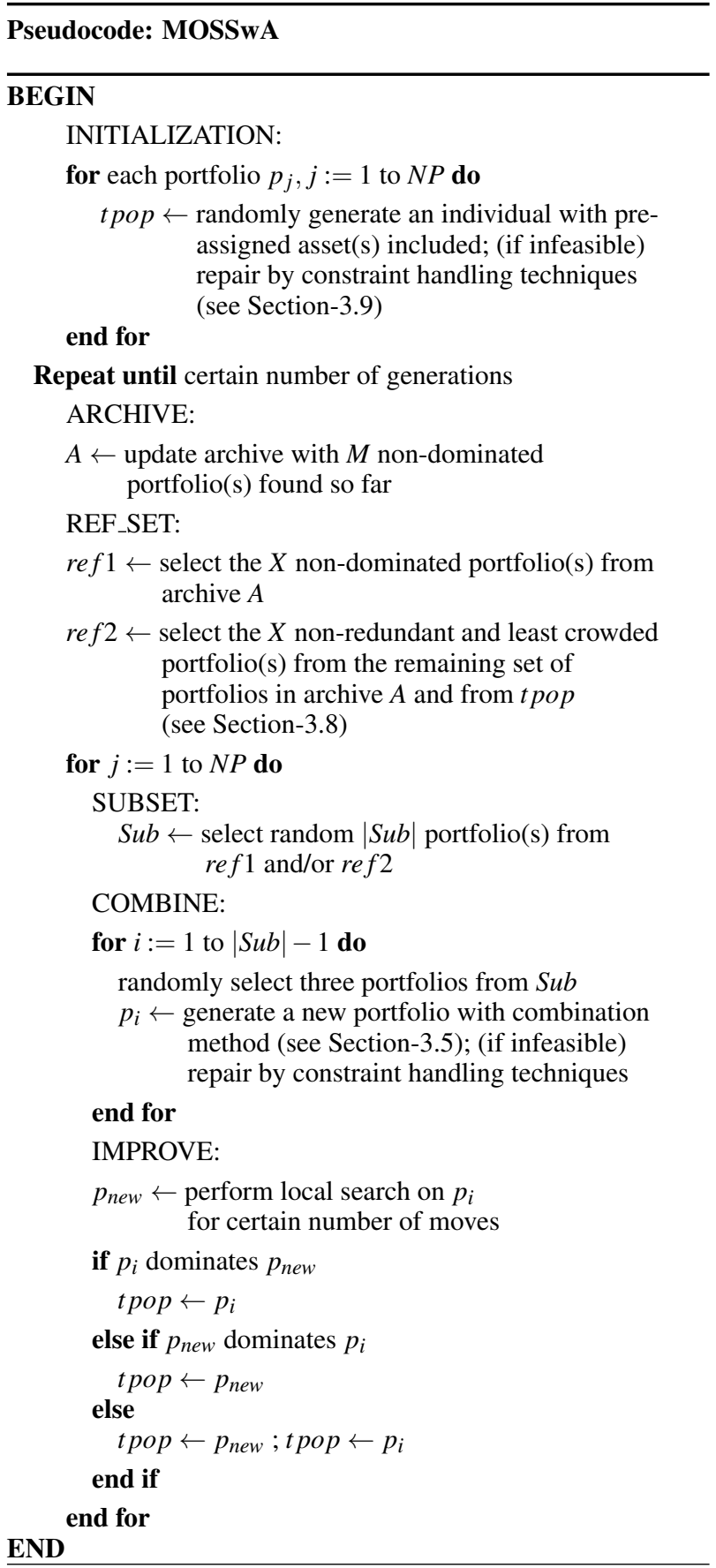

Figure 1: Pseudocode of the proposed MOSSwA

subset $(S u b)$ will later be used in solution combination method to produce new solutions. Our approach generates the subset by three different variants as follows:

1. randomly select $X$ solutions from $\operatorname{ref} 1$.

2. randomly select $X$ solutions from $r e f 2$. 
3. randomly select arbitrary $R$ solutions from ref 1 and $X-R$ solutions from ref 2 where $R \leq X$.

\subsection{Solution Combination Method}

This method uses the generated subset Sub (see Section-3.4) and combines solutions from $S u b$ and returns one or more trial solutions. In finance literature, it is considered to be a fundamental premise to utilize assets that have low correlation with each other. Hence the assets which are less correlated to each other are preferable to the heavily correlated assets. It is also commonly believed that it is beneficial to reduce the portfolio's standard deviation of return. Intuitively, investors prefer higher return assets with less risk.

By taking into accounts of the above stated intuitive appeal, in this work, we propose three problemspecific combination mechanisms for the constrained portfolio selection problem as follows: Three portfolios ( $p 1, p 2$ and $p 3)$ from subset $S u b$ are selected to use in the combination mechanism. The assets selected in these three portfolios are observed and analysed. The set potential is constructed by inserting all the indexes of assets which are selected in at least two out of the three portfolios. A new portfolio is then constructed by selecting random $n$ indexes of assets in potential set where $n \leq K$. Like the DE offspring generation scheme investigated in (Robič and Filipič, 2005), the proportion $w$ of those selected assets for the new solution are assigned as follows:

$$
w_{i}:=w 3_{i}+r[0,1] \times\left(w 1_{i}-w 2_{i}\right)
$$

The remaining assets of the new portfolio are selected by one of the following heuristic methods in selection of asset:

HigherExpRet: $\mathrm{K}$ - $\mathrm{n}$ assets which have the highest expected return values are selected in the new solution and the proportions of those selected assets are randomly assigned.

LessSTD: $\mathrm{K}$ - $\mathrm{n}$ assets which have the least standard deviation values are selected in the new solution and the proportions of those selected assets are randomly assigned.

LeastCov: $\mathrm{K}$ - $\mathrm{n}$ assets which have the lowest correlation values are selected in the new solution and the proportions of those selected assets are randomly assigned.

It may be noted that the combination mechanisms construct solutions that may or may not be feasible. Hence, the repair mechanism is employed if the newly generated solution violates the constraints. (see Section-3.9)

\subsection{Improvement Method}

The solutions obtained by the combination method (see Section-3.5) are improved by a local search technique (i.e., hill climbing). It simply moves the current solution to a better candidate solution by using mutation operation. This is done by randomly altering a proportion of an asset or a selection of the asset in the portfolio until no improved solutions can be found in a certain number of moves. Then the trial population is updated by adding those non-dominated solutions.

\subsection{Maintaining the External Archive}

The main objective of the external archive $(A)$ is to keep all the non-dominated solutions encountered along the search process. This approach is adopted in order to save and update all well spread nondominated solutions generated by the algorithm during the search.

In each generation, the archive $(A)$ is updated with the non-dominated solutions from the trial population. The computational time of maintaining the archive increases with the archive size. Hence, the size of the archive is restricted to a prespecified value. When the external archive has reached its maximum capacity $(M)$, the crowding distances of the solutions are calculated to delete the most crowded archive members.

\subsection{Reference Set Update Method}

Once the archive $(A)$ has been updated with nondominated solutions obtained from the trial population (tpop), the reference sets are updated. For our proposed algorithm for the portfolio optimization problem, the reference set update method has been devised as follows.

The non-dominated solutions obtained from the archive $(A)$ are sorted by using the Pareto dominance. The $X$ best non-dominated solutions obtained from the archive $(A)$ are updated to refl. Similarly, the solutions in the trial population are sorted by the crowding distance. The $X$ least crowded solutions obtained from the sorted archive $(A)$ and trial (tpop) solutions are updated to ref2.

\subsection{Constraint Handling}

During the population sampling, each constructed individual must be repaired if the representative portfolio does not satisfy the constraints of the problem. The budget constraint in Eq.(3) is satisfied by firstly normalizing the weights: $w_{i}=w_{i} / \sum_{j=1}^{N} w_{j}$ over those assets selected. Moreover, the bounding constraint 
in Eq.(5) requires the proportion of asset $i$ to be in the range $\left[\varepsilon_{i}, \delta_{i}\right]$. If the proportion of asset after the normalization violates the quantity constraints, then it employs repair mechanism described in (Chang et al., 2000).

\section{EXPERIMENTAL RESULTS}

In this work, we compared the performance of the proposed algorithm with the Non-dominated Sorting Genetic Algorithm (NSGA-II) (Deb et al., 2002), the Strength Pareto Evolutionary Algorithm (SPEA2) (Zitzler et al., 2001) and Pareto Envelope-based Selection Algorithm (PESA-II) (Corne et al., 2001).

Twenty independent runs were performed for all experiments and the same random seed is assigned to each set of the sum so that the algorithms start with the same initial population. In order to ensure a fair comparison, we have used the same population size and archive size (if applicable) for all the algorithms tested in this work. We have chosen to run all the algorithms for the same stopping criteria (i.e. the same number of evaluations) to generate the Pareto front. Each algorithm also uses the same encodings (see Section-3.1) and repair mechanism (see Section-3.9) when a newly constructed portfolio violates the considered constraints. NSGA-II uses binary tournament selection based on the crowding distance. NSGA-II, SPEA2 and PESA-II use simulated binary crossover and polynomial mutation evolutionary operators.

Before the experiments were performed, parameters are tuned for all algorithms using the smallest problem instance, i.e. Hang Seng. The parameter value of the compared algorithms are provided in Table-1. For constraint values, we use $K=10$, $\varepsilon_{i}=0.01(i=1, \ldots, N), \delta_{i}=1.0(i=1, \ldots, N)$ and $z_{i}=$ $1(i=30)$.

Table 1: Parameter setting of for four algorithms.

\begin{tabular}{lllll}
\hline Parameters & MODEwAwL & NSGA-II & SPEA2 & PESA-II \\
\hline Number of Population $(N P)$ & 100 & 100 & 100 & 100 \\
Number of Generation & $10,000 \mathrm{~N}$ & $10,000 \mathrm{~N}$ & $10,000 \mathrm{~N}$ & $10,000 \mathrm{~N}$ \\
Scaling Factor $(F)$ & 0.3 & - & - & - \\
Crossover Probability $(C R)$ & 0.9 & 0.9 & 0.9 & 0.9 \\
Crossover Distribution Index & - & 20 & 20 & 20 \\
Mutation Probability & - & $1 / \mathrm{N}$ & $1 / \mathrm{N}$ & $1 / \mathrm{N}$ \\
Mutation Distribution Index & - & 20 & 20 & 20 \\
Tournament Round & - & - & 1 & - \\
Number of Bisection & - & - & - & 5 \\
Archive Size $(M)$ & 100 & - & 100 & 100 \\
\hline
\end{tabular}

\subsection{Dataset}

A test data for the portfolio optimization problems from the OR-library (Beasley, 1990) is used to evaluate the performance of the algorithms described above. These datasets contain the estimated returns and the covariance matrix of five different stock market indices: Hang Seng in Hong Kong, DAX 100 in Germany, FTSE 100 in UK, S\&P 100 in

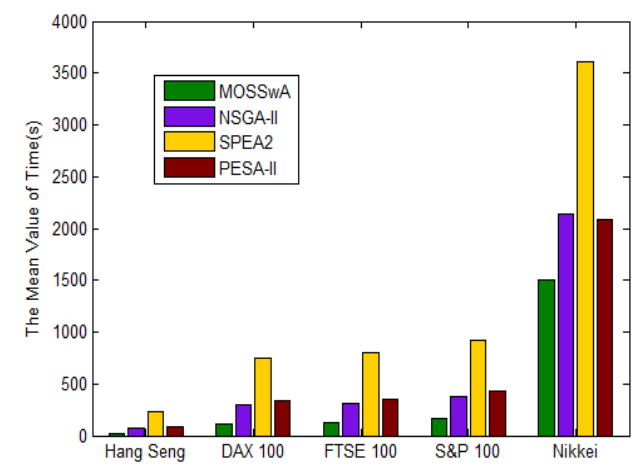

Figure 7: Running Time Measures of the algorithms for Constrained Portfolio Optimization Problem.

USA and Nikkei 225 in Japan. For each set of the test data, the number of assets $N$ is $31,85,89$, 98 and 225, respectively. In the current literature of portfolio selection problem, this set of dataset has been widely tested, and is recognized as the benchmark to evaluate computational algorithms. All information of the dataset itself and their best known solutions can be accessed online at the URL http://people.brunel.ac.uk/ mastjjb/jeb/orlib/portinfo .html.

\subsection{Performance Metric}

To evaluate the performance of the proposed algorithm for the constrained portfolio optimization problem, we use three criteria: Inverted Generational Distance, Generational Distance and Diversity metric $(\Delta)$.

\subsubsection{Inverted Generational Distance (IGD)}

The Inverted Generational Distance (Sierra and Coello, 2005) uses the true Pareto front as a reference and measures the distance from the true Pareto front to the Pareto front obtained by an algorithm. It is mathematically defined as:

$$
I G D=\frac{\sqrt{\sum_{i=1}^{Q} d_{i}^{2}}}{Q}
$$

where $Q$ is the number of solutions in the true Pareto front and $d_{i}$ is the Euclidean distance between each of the solution and the nearest member from the set of non-dominated solutions found by the algorithm. This metric measures both the diversity and the convergence of an obtained non-dominated solution set. The smaller the value of this metric, the closer the obtained front is to the true Pareto front. 

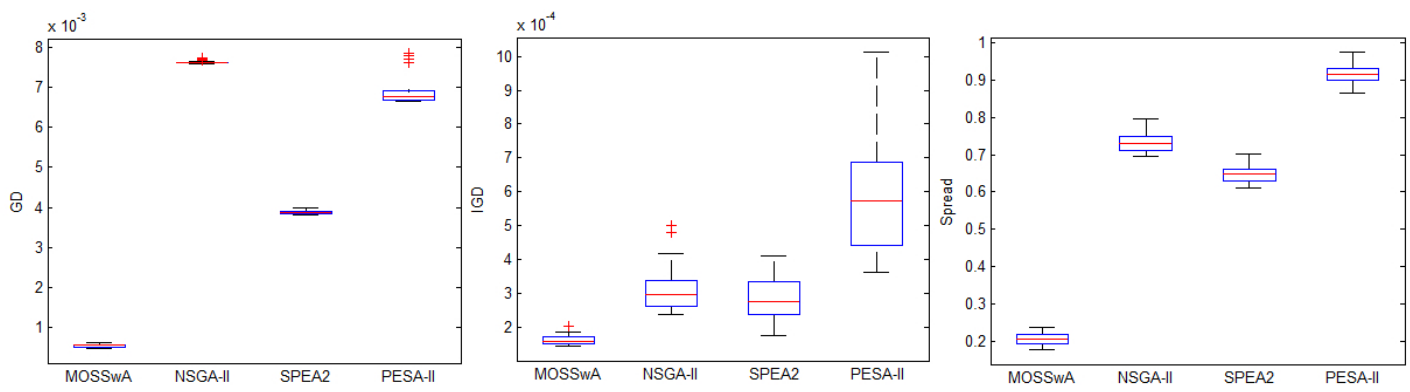

Figure 2: Performance Comparisons of the algorithms in term of GD, IGD and $\Delta$ metrics for Hang Seng.
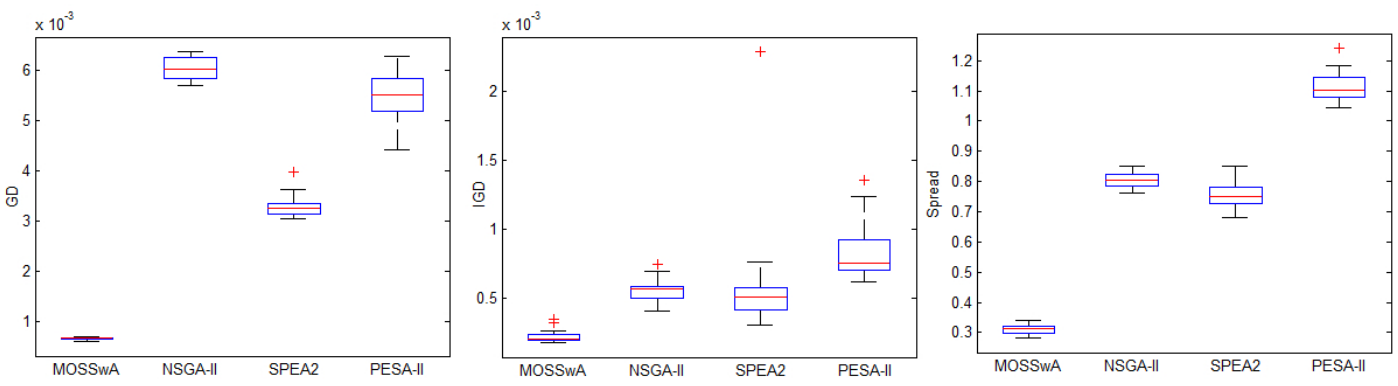

Figure 3: Performance Comparisons of the algorithms in term of GD, IGD and $\Delta$ metrics for DAX 100.
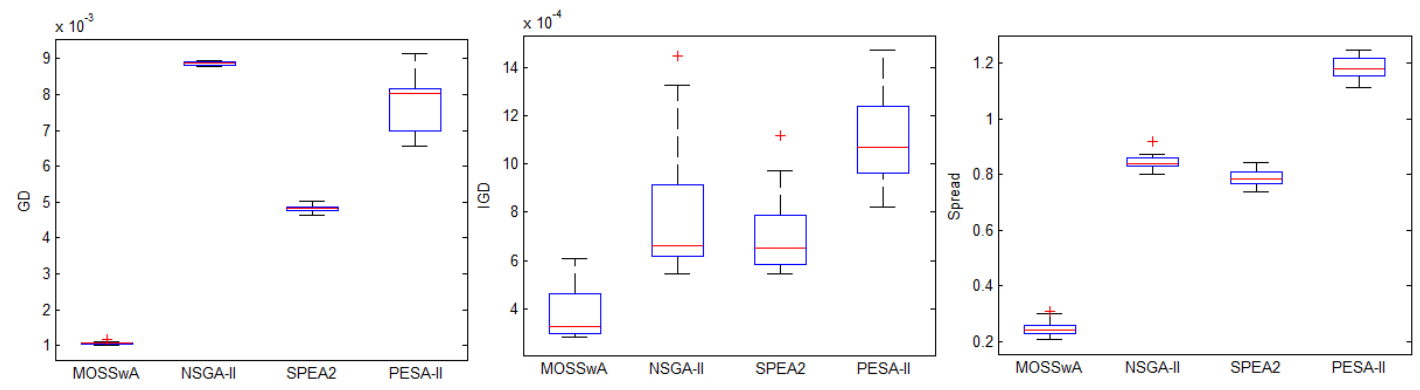

Figure 4: Performance Comparisons of the algorithms in term of GD, IGD and $\Delta$ metrics for FTSE 100.
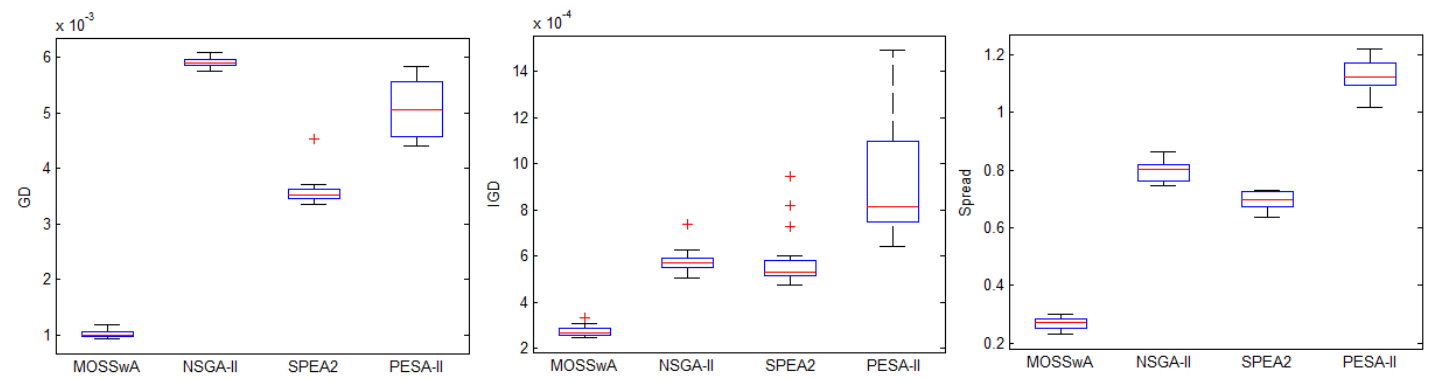

Figure 5: Performance Comparisons of the algorithms in term of GD, IGD and $\Delta$ metrics for S\&P 100.

\subsubsection{Generational Distance (GD)}

The Generational Distance (Van Veldhuizen and Lamont, 1998) is a variant of the IGD. It measures how far the solutions of the computed Pareto front obtained by an algorithm are from those at the true Pareto front. The smaller value indicates that all the generated solutions are on the true Pareto front. 

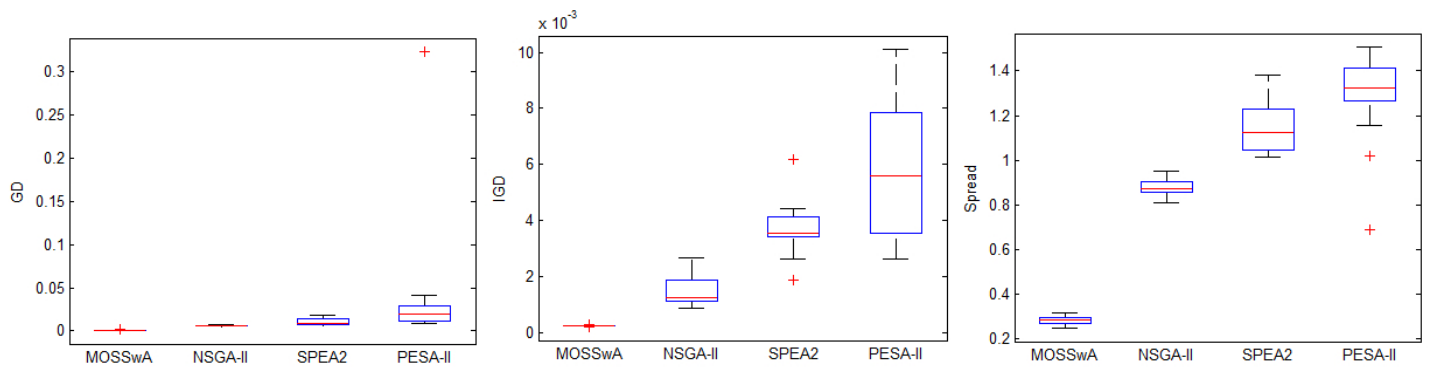

Figure 6: Performance Comparisons of the algorithms in term of GD, IGD and $\Delta$ metrics for Nikkei.
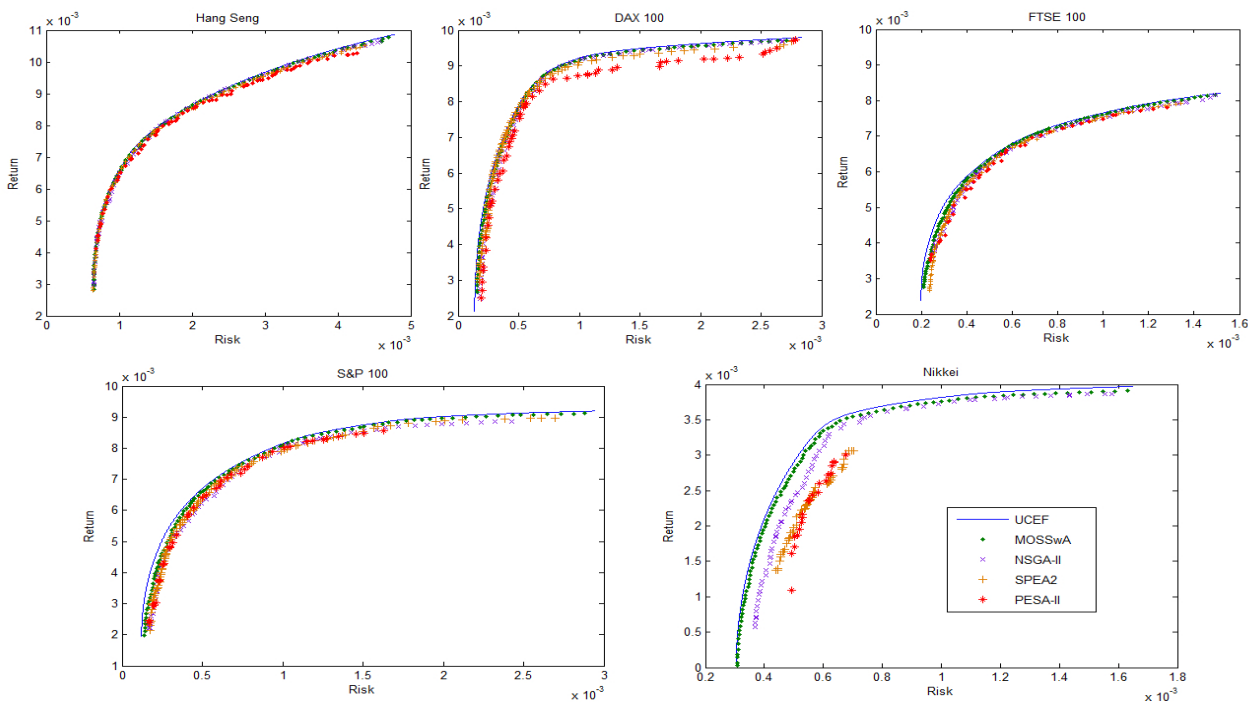

Figure 8: Comparison of Obtained Efficient Frontier of all the algorithms for Constrained Portfolio Optimization Probelm.

\subsection{3 $\operatorname{Spread}(\Delta)$}

The Diversity metric $(\Delta)$ (Deb et al., 2002) measures the extent of spread achieved among the nondominated solutions as follows:

$$
\Delta=\frac{d_{f}+d_{l}+\sum_{i=1}^{|Q|-1}\left|d_{i}-\bar{d}\right|}{d_{f}+d_{l}+(|Q|-1) \bar{d}}
$$

where $d_{i}$ is the Euclidean distance in the objective space between consecutive solutions in the obtained non-dominated front $Q$, and $\bar{d}$ is the average of these distances. The parameters $d_{f}$ and $d_{l}$ are the Euclidean distance between the extreme solutions of the Pareto front $P^{*}$ and the boundary solutions of the obtained front $Q$. The lower value of the spread $(\Delta)$ indicates a better diversity.

\subsubsection{Comparisons of the algorithms}

The experimental results of GD, IGD and $\Delta$ of the four algorithms performed on five datasets from
OR-library (see Section-4.1) are shown in Figure2, Figure-3, Figure-4, Figure-5 and Figure-6. The running time of the algorithms are shown in Figure7. The results showed that our proposed algorithm (MOSSwA) is not only superior in performance measures but also is efficient in computational time compared with NSGA-II, SPEA2 and PESA2.

For illustrative purpose, four obtained efficient frontiers of the algorithms from a single run for five problem instances along with the true unconstrained efficient frontier (UCEF) are provided in Figure-8.

To further support our observation that MOSSwA outperforms others, we compare the IGD values of the five algorithms by using Student's t-test (Walpole et al., 1998). The statistical results obtained by a two-tailed t-test with 38 degrees of freedom at a 0.05 level of significance are given in Table-[2]. The result of Algorithm-1 $\leftrightarrow$ Algorithm-2 is shown as "+", "-", or " " when Algorithm-1 is significantly better than, significantly worse than, or statistically equivalent to Algorithm-2, respectively. Results show that MOSSwA outperforms other algorithms in all prob- 
Table 2: Student t-Test Results of Different Algorithms on five problem instances from OR-Library.

\begin{tabular}{|l|l|l|l|l|l|}
\hline Algorithm1 $\leftrightarrow$ Algorithm2 & Hang Seng & DAX 100 & FTSE 100 & S \& P 100 & Nikkei \\
\hline MOSSwA $\leftrightarrow$ NSGA-II & + & + & + & + & + \\
\hline MOSSwA $\leftrightarrow$ SPEA2 & + & + & + & + & + \\
\hline MOSSwA $\leftrightarrow$ PESA-II & + & + & + & + & + \\
\hline NSGA-II $\leftrightarrow$ SPEA2 & $\sim$ & $\sim$ & $\sim$ & $\sim$ & + \\
\hline NSGA-II $\leftrightarrow$ PESA-II & + & + & + & + & + \\
\hline SPEA2 $\leftrightarrow$ PESA-II & + & + & + & + & + \\
\hline
\end{tabular}

lem instances. We therefore can conclude that the proposed MOSSwA has the best optimization performance for the portfolio optimization problem with considered constraints.

\section{CONCLUSIONS}

In this work, we presented a multi-objective evolutionary algorithm based on Scatter Search with external archive (MOSSwA) for solving the meanvariance portfolio selection problem with cardinality, quantity and pre-assignment constraints. Experimental results indicate that the proposed adapted multiobjective Scatter Search algorithm (MOSSwA) outperforms the Non-dominated Sorting Genetic Algorithm (NSGA-II), Strength Pareto Evolutionary Algorithm (SPEA-2) and Pareto Envelope-based Selection Algorithm (PESA-II) in all performance measures. The proposed hybrid metaheuristic algorithm follows the basic structure of the Scatter Search and defines the reference set solutions with the Pareto dominance and crowding distance concepts. New subset generation and combination methods are proposed to contribute to the literature in order to generate efficient and diversified portfolios. When cardinality and preassignment constraints are considered, the proposed three combination mechanisms enhance the solution quality significantly in terms of both the computational time and all the measures of solution quality.

\section{REFERENCES}

Beasley, J. (1990). Or-library: Distributing test problems by electronic mail. Journal of the Operational Research Society, 41(11):1069-1072.

Branke, J., Scheckenbach, B., Stein, M., Deb, K., and Schmeck, H. (2009). Portfolio optimization with an envelope-based multi-objective evolutionary algorithm. European Journal of Operational Research, 199(3):684-693.

Chang, T., Meade, N., Beasley, J., and Sharaiha, Y. (2000). Heuristics for cardinality constrained portfolio optimisation. Computers and Operations Research, 27(13):1271-1302.

Corne, D. W., Jerram, N. R., Knowles, J. D., Oates, M. J., et al. (2001). Pesa-ii: Region-based selection in evolutionary multiobjective optimization. In Proceedings of the Genetic and Evolutionary Computation Conference (GECCO2001. Citeseer.
Deb, K., Pratap, A., Agarwal, S., and Meyarivan, T. (2002). A fast and elitist multiobjective genetic algorithm: Nsga-ii. Evolutionary Computation, IEEE Transactions on, 6(2):182-197.

Fonseca, C. and Fleming, P. (1995). An overview of evolutionary algorithms in multiobjective optimization. Evolutionary computation, 3(1):1-16.

Glover, F., Laguna, M., and Martí, R. (2000). Fundamentals of scatter search and path relinking. Control and cybernetics, 39(3):653-684.

Lwin, K. and Qu, R. (2013). A hybrid algorithm for constrained portfolio selection problems. Applied Intelligence,DOI:10.1007/s10489-012-0411-7.

Markowitz, H. (1952). Portfolio selection. The Journal of Finance, 7(1):pp. 77-91.

Markowitz, H. (1959). Portfolio selection: Efficient diversification of investments. John Wiley and Sons, New York.

Moral-Escudero, R., Ruiz-Torrubiano, R., and Suarez, A. (2006). Selection of optimal investment portfolios with cardinality constraints. In Evolutionary Computation, 2006. CEC 2006. IEEE Congress on, pages 2382-2388. IEEE.

Robič, T. and Filipič, B. (2005). Demo: Differential evolution for multiobjective optimization. In Evolutionary Multi-Criterion Optimization, pages 520-533. Springer.

Sierra, M. R. and Coello, C. A. C. (2005). Improving pso-based multi-objective optimization using crowding, mutation and epsilon-dominance. In EMO'05, pages 505-519.

Skolpadungket, P., Dahal, K., and Harnpornchai, N. (2007). Portfolio optimization using multi-obj ective genetic algorithms. In Evolutionary Computation, 2007. CEC 2007. IEEE Congress on, pages 516-523. IEEE.

Van Veldhuizen, D. A. and Lamont, G. B. (1998). Multiobjective evolutionary algorithm research: A history and analysis. Technical report, Citeseer.

Walpole, R. E., Myers, R. H., Myers, S. L., and Ye, K. (1998). Probability and statistics for engineers and scientists, volume 8. Prentice Hall Upper Saddle River, NJ:.

Zitzler, E., Laumanns, M., Thiele, L., Zitzler, E., Zitzler, E., Thiele, L., and Thiele, L. (2001). Spea2: Improving the strength pareto evolutionary algorithm. 